\title{
A nonparametric approach for evaluating long-term energy policy scenarios: an application to the Greek energy system
}

\author{
George Halkos, Nickolaos G Tzeremes ${ }^{*}$ and Panayiotis G Tzeremes
}

\author{
* Correspondence: bus9nt@uth.gr \\ Laboratory of Operations Research, \\ Department of Economics, \\ University of Thessaly, Korai 43, \\ 38333, Volos, Greece
}

\begin{abstract}
This paper by using Long-range Energy Alternatives Planning System (LEAP) constructs four different renewable energy scenarios for the Greek transport, energy, and industry sectors. By projecting the demand for renewable energy and the associated resulting carbon dioxide emissions up to the years 2020 and 2030, the paper applies in a second stage data envelopment analysis (DEA) evaluating the Greek renewable energy policy. As a result, it provides a quantitative measure for future renewable energy policy evaluation under different renewable energy consumption scenarios. The results reveal that the main challenge for the Greek policy makers will be the energy policies associated with the renewable energy usage of the Greek industry since they are rigid toward the adoption of technologies utilizing renewable energy sources. It appears that under the four different energy policy scenarios, the Greek industry sector will not be able to meet its renewable energy targets set by the Greek government. Finally, the analysis reveals that the renewable energy targets set for 2020 and 2030 can be met for the energy sector. However, the renewable energy targets set for the transport sector can only be met for the year 2030.

JEL classifications: C60; Q47; Q53; Q58

Keywords: Climate change; Renewable energy sources; Greek energy system; Linear programming; Nonparametric analysis
\end{abstract}

\section{Background}

Long-range Energy Alternatives Planning System (LEAP) is a widely used software tool for energy policy analysis and climate change mitigation assessment developed by the Stockholm Environment Institute. According to Heaps (2008), LEAP is a standard tool that enables countries to contact integrated resource planning, greenhouse gases (GHG), mitigation assessments, and low-emission development strategies (LEDS). Furthermore, several countries have been based on LEAP's output in order to report to the United Nations Framework Convention on Climate Change (UNFCCC).

There are various studies in Greece that have been conducted in order to provide the literature with long-term projections in the energy sector using LEAP (among others, Papagiannis et al. 2008; Giatrakos et al. 2009; Roinioti et al. 2012). According to Bhattacharyya and Timilsina (2010), LEAP is based on the accounting framework in order to generate energy demand (and supply) and on the physical description of the

(c) 2015 Halkos et al. This is an Open Access article distributed under the terms of the Creative Commons Attribution License (http://creativecommons.org/licenses/by/4.0), which permits unrestricted use, distribution, and reproduction in any medium, provided the original work is properly credited. 
examined energy system. Furthermore, on their extensive review Bhattacharyya and Timilsina (2010) emphasize the fact that LEAP is based on the scenario approach in order for several paths of energy system evolution to be developed. According to Heaps (2002), the forecast of the energy demand is based on the effect of alternative market shares, whereas the supply side is based on a what-if analysis and possible development scenarios which LEAP integrates through simulation and accounting approaches.

Our paper constructs four different scenarios for the period 1990-2030 in order to evaluate the demand of Greek energy derived from renewable energy sources (RES) and the GHG emissions generated over the same period for the sectors of industry, transport, and energy. Therefore, in the first stage, the paper forecasts the energy demand derived from renewable sources alongside the generated GHG emissions (under the four scenarios). Furthermore, in the second stage analysis, it applies a nonparametric estimator based on the mathematical approach known as data envelopment analysis (DEA $)^{1}$ in order to evaluate the efficiency of the Greek renewable energy policies imposed under the Law L3851/2010 which was introduced in order to comply with the European targets set in 2007 and in $2014 .^{2}$ As a result, the paper presents a way for evaluating the efficiency of the future implementation of renewable energy policies, which in our case have been set by the Greek government and under four different energy-based scenarios.

The article is constructed as follows. Section 2 presents a review of the relative literature, the four scenarios for the Greek energy sector and the nonparametric methodology adopted. Section 3 presents the empirical findings, whereas, the last section concludes the paper.

\section{Methods}

\subsection{A Brief review of LEAP-based studies}

The mitigation of greenhouse gases has received much attention since the ratification of Kyoto Protocol in 1992 at the United Nations Rio Earth Summit. The fulfillment of this target requires a shift toward cleaner energy, renewable energy sources, energy efficiency, and low-carbon options. The construction and examination of alternative scenarios is important for the decision maker (countries, international parties, ministries, etc.) in order to decide the best path to follow. Long-range Energy Alternatives Planning System software is an excellent and widely used tool for scenario analysis. LEAP has been widely used in various sectors such as energy and electricity, industry, and transport.

Bala (1997) used LEAP to investigate the rural energy system of Bangladesh which is based primarily on biomass. The author argued against massive deforestation as a solution to the energy problem. Islas et al. (2003) examined the Mexican energy sector. They constructed scenarios for alternative energy sources such as natural gas and RES and found that in most cases, alternative scenarios are better than the oil-based baseline scenario. Ghanadan and Koomey (2005) constructed four scenarios about the energy sector in California. The alternative scenarios are based on cleaner energy and RES, behavioral changes, and energy efficiency changes. Shin et al. (2005) assessed the use of landfill gas as a renewable energy source for electricity in Korea. The results are not clear due to economic, technological, and regulatory uncertainties of landfill gas. Mulugetta et al (2007) investigated the power sector in Taiwan for the time period 2002-2020. They constructed a business-as-usual and two alternative scenarios about 
improvements in efficiency and introduction of RES such as biomass, solar, wind, and hydropower.

The case of the Chinese electricity sector was investigated by Zhang et al. (2007). Alternative policies were examined such as the replacement of coal with nonfossil fuels and RES and the incorporation of new advanced technology. Cai et al. (2008) studied five sectors of the Chinese economy (electricity, iron and steel, cement, pulp and paper, and transport). The results indicated that current mitigation options should be further encouraged, among others with structure reformation at the sector level, energy efficiency options, RES, and mitigation incentives. Dagher and Ruble (2011) constructed scenarios about the electricity sector in Lebanon and examined the shift toward natural gas in one scenario and toward RES in another scenario. Yophy et al. (2011) investigated the energy sector in Taiwan. They constructed scenarios about the mitigation of $\mathrm{CO}_{2}$ emissions using various assumptions such as enhancing energy efficiency, the closing of existing nuclear plants, and the possible negative effects from economic growth. Park et al. (2013) examined the transition of the Korean electricity sector toward RES. The alternative scenarios assume different levels of environmental policies. The only scenario which yielded reduced emissions was the sustainability scenario. This scenario assumes that RES would generate the most electricity by 2050 .

Another important cross-national attempt has been made from the European Union toward the reduction of GHG emissions known as the "20-20-20" targets. According to these targets, countries are committed to reduce the total EU GHG emission by $20 \%$ in 2020 compared to the 1990 levels. The parties are also obliged to raise the share of RES to $20 \%$ of the total EU energy consumption and to improve the EU's energy efficiency by $20 \%$. The implementation of these ambitious targets has brought scenario analysis into the center of the scientific community's attention. The construction of the alternative scenarios and the results of the analysis are crucial for the decision maker in order to decide which path will follow toward the fulfillment of the targets.

There are not many LEAP studies for Greece. Giatrakos et al. (2009) investigated the RES in the power sector at the island of Crete, using a business-as-usual scenario and two alternative scenarios. The alternatives assume different penetration levels for the demand-side management policies. The results indicate that the EU targets are achievable by the end of 2015 without compromising the economic growth of the island. In fact, the results indicate that the targets could be surpassed, leading to a 30\% RES penetration and $50 \%$ GHG reduction by 2030 . The outcome is a result of wind and photovoltaic parks which are expected to be constructed at Crete. Karapidakis et al. (2011) have also studied the power sector in Crete Island toward 2020 using two alternative scenarios. The first scenario assumes an increase of RES to $20 \%$ and a moderate increase in the total installed capacity wind and photovoltaic plants. The second scenario assumes a significantly larger increase of RES to 50\%, the installation of hydro-pumped storage systems and also a considerable increase in the total installed capacity of wind and photovoltaic plants. The results indicated stable emissions for the first scenario and $40 \%$ lower emissions for the second scenario.

Roinioti et al. (2012) examined the Greek energy system with a focus on electricity production system. The authors used LEAP and created a business-as-usual scenario and four alternative scenarios based on uncertainties and growth assumptions. Specifically, the first alternative scenario is the green scenario which assumes low emissions and high 
growth rate, with the introduction of advanced RES technologies. The orange scenario assumes high emissions and high growth rate, the red scenario assumes high emissions and low growth rate, and the blue scenario assumes low emissions and low growth rate. The results indicated that from an environmental point of view, the best is the green scenario and the worst is the orange scenario. In addition, green is the only scenario to achieve the $40 \%$ RES target in electricity production by 2020 . Ikonomopoulos et al. (2013) investigated the greenhouse gas emissions in the Greek electricity sector by 2030. The authors created a reference scenario and three alternatives. The reference scenario assumes that old power stations will close and new RES stations will open according to the announcements and time schedule of the Ministry of Environment. The first alternative scenario assumes delays in closing of old thermal stations and opening new advanced ones. The second alternative scenario assumes the connection of islands to the mainland grid, and the last scenario assumes the introduction of hard coal in Greek power generation. The results revealed that all four scenarios reduce emissions and highlighted the key role of RES.

Apart from the energy and electricity sector, there are a number of studies using LEAP on the Greek hotel and building sector. Maleviti et al. (2012) studied the energy consumption in Greek four- and five-star hotels. The authors constructed a businessas-usual scenario and an alternative scenario which assumes the implementation of a restrictive policy framework toward the reduction of energy consumption, the improvement of energy efficiency, and other improvements. The outcome of this study indicated the importance of diffusing the information about potential energy improvements to hotel managers. Katsigiannis et al. (2012) investigated the effects of retrofitting buildings to the power system of Crete Island which is not connected to the mainland power grid of Greece. The authors constructed eight alternative scenarios with respect to energy and electricity consumption and RES penetration emphasizing of RES technologies for the Greek energy policy.

\subsection{A description of the LEAP-based renewable energy policy scenarios ${ }^{3}$}

Scenarios are based on future energy systems which are constructed based on specific economic condition trends and possibilities (Wiseman et al. 2011; Carter 2007; Moss et al. 2010). The main concept of LEAP is an end-use driven scenario analysis with a baseline scenario and different other alternative scenarios. The scenarios are used for a number of "what if" questions under the arrangement of user-defined assumptions. The set of conditions is detailed in the scenarios and is constructed in order to encompass some factors (parameters) that are anticipated to change.

In our case, there are four scenarios generated under different options. The policy options and the key assumptions the scenarios are based on are presented next. ${ }^{4}$

\subsubsection{Baseline scenario-BASE, BAU}

The first scenario is the "Baseline", which is based on historical trends from 1990 till 2010. Changes in demographic and macroeconomic variables are given in Table 1 . Specifically, Table 1 describes the projections for the annual population growth rate, annual GDP growth rate, annual growth rate of income, and annual growth rate of GDP per capita till the target year 2030 (Ministry of Environment, Energy and Climate 
Table 1 Changes of demographic and macroeconomic variables used

\begin{tabular}{llllllll}
\hline & $\mathbf{2 0 1 1}$ & $\mathbf{2 0 1 2}$ & $\mathbf{2 0 1 3}$ & $\mathbf{2 0 1 4}$ & $\mathbf{2 0 1 5}$ & $\mathbf{2 0 1 6}$ & $\mathbf{2 0 1 7}$ \\
\hline Annual population growth rate (\%) & 0.1 & 0.0 & -0.2 & -0.2 & -0.1 & -0.3 & -0.2 \\
Annual GDP growth rate (\%) & -4.0 & -2.6 & 1.1 & 2.1 & 2.1 & 2.6 & 2.6 \\
Annual growth rate of income (\%) & -4.0 & -3.7 & 0.8 & 2.8 & 2.5 & 2.6 & 2.6 \\
Annual growth rate of GDP per capita (\%) & -9.0 & -2.0 & 0.9 & 1.8 & 1.6 & 2.6 & 2.6 \\
& 2018 & 2019 & 2020 & 2025 & 2030 & & \\
Annual population growth rate (\%) & -0.1 & -0.1 & -0.2 & -0.2 & -0.4 & \\
Annual GDP growth rate (\%) & 2.5 & 2.5 & 2.9 & 2.2 & 1.5 & \\
Annual growth rate of income (\%) & 2.5 & 2.5 & 2.9 & 2.2 & 1.5 & & \\
Annual growth rate of GDP per capita (\%) & 2.5 & 2.5 & 2.9 & 2.2 & 1.5 & & \\
\hline
\end{tabular}

Projections are based on estimates by the Ministry of Environment, Energy and Climate Change (2013).

Change 2013). The projected potential withdrawals of power plants may be found in Halkos et al. (2014).

\subsubsection{Target 2020 scenario-TAR20}

The second scenario is based on the European target set in 2007, in order to develop an energy-efficient and low-carbon Europe via an increase in the share of the EU energy consumption produced from renewable resources to $20 \%$. According to the government and to the Law L3851/2010, it is stated that the protection of the climate or the reduction of GHG emissions, through the promotion of electrical energy production from RES, is a crucial element of the energy sector of the country. In order to achieve the national target of $20 \%$ contribution of the energy produced from RES to the gross final energy consumption, specific targets include increasing RES electricity share by $40 \%$, RES heating and cooling share for the household sector by $20 \%$, and RES transport share by $10 \%$. This target will be achieved through the large penetration of RES technologies in electricity production, heat supply, and in the transport sector.

The changes in the demographic and macroeconomic variables that are used in the target 2020 scenario are also presented in Table 1. Finally, we assume a 50\% increase of RES capacity, which corresponds to 5311.7 MW. Specifically, as the Hellenic Transmission System Operator S.A. publishes binding and final offers for connection system or network for power stations of renewable energy and stations and cogeneration plants of electricity and heat and high performance (CHP), we assume that till 2020, half of the nonbinding offers will be achieved. Table 2 describes in details the structure of the assumed generated capacity per RES category.

\subsubsection{Target 2030 scenario-TAR30}

We follow the target set in 22 January 2014 by the European Commission toward a renewable energy economy. Specifically, the share of renewable energy penetration in final consumption is set to increase at least up to $27 \%$ by 2030 . This will be achieved by the introduction of RES in industry. Following Heaps et al. (2009) concerning the industry sector, $\mathrm{CO}_{2}$ emissions can be further reduced through the increased use of biomass, natural gas, and increased participation of RES in electricity, the iron and 
Table 2 Generation capacity projections per RES category till 2020 and 2030

\begin{tabular}{lll}
\hline RES & Capacity (MW) $\mathbf{2 0 2 0}$ & Capacity (MW) $\mathbf{2 0 3 0}$ \\
\hline Photovoltaics & 207.5 & 415 \\
Wind park & 4666.5 & 9333 \\
Small hydro & 350.2 & 640.2 \\
Biomass & 87.5 & 175 \\
Total & 5311.7 & $10,563.2$ \\
\hline
\end{tabular}

Source: Hellenic Transmission System Operator S.A. available http://www.desmie.gr/ape-sithya/stathmoi-ape-sithya-meprosfora-syndesis/.

steel production sector, the cement production, the chemical production, and other industrial subsectors. As far as the changes in demographic and macroeconomic variables that are used in the target 2030 scenario, these are given also in Table 1. Furthermore, we assume a 100\% increase of RES capacity, which corresponds to 10,563.2 MW. Specifically, as in the previous scenario and relying on the Hellenic Transmission System Operator S.A., the last column of Table 2 describes in details the structure of the assumed generated capacity per RES category.

\subsubsection{Green scenario-green}

Under this scenario, we follow as in TAR30 the target set in 22 January 2014 by the European Commission toward a renewable energy economy. However, and in contrast to TAR30, we assume that the share of renewable energy penetration in final consumption is set to increase at least up to $27 \%$ by 2020 instead of 2030. The same assumptions imposed for TAR30 are also imposed for the green scenario; however, under the green scenario, the Greek government should increase the share of energy consumption produced from renewable resources to $27 \%$ by 2020 .

Figure 1 presents the projections of GHG emissions for the sectors of industry, transport, and energy. As can be observed, industry produces the lowest levels of GHG emissions, whereas the transport sector produces the highest GHG emission levels. As can be viewed, the emissions produced by the Greek industry have been declined especially during the financial crisis period. The same is reported for the energy sector. However, the emissions generated by the transport sector have been monotonically increasing (Base and TAR20 scenarios). In all cases as expected and under the base scenario, the sectors will be generating higher levels of GHG emissions compared to the green scenario.

Finally, Figure 2 presents the estimated energy consumption from RES under the four scenarios. It can be viewed that under the green scenario, the different sectors will have more investments on RES and therefore the consumption levels will be higher. However, again it can be noticed that the energy levels generated from RES of the industry sector (Figure 2a) will be significantly lower compared to the sectors of transport (Figure 2b) and energy (Figure 2c).

\subsection{Data Envelopment Analysis}

In order to evaluate the efficiency of the Greek government's energy policies, we need to evaluate also their ability to reduce greenhouse gases under the four renewable energy policy scenarios described previously (BAU, TAR20, TAR30, and green). Specifically, we need to evaluate under the four scenarios generated in LEAP for the period 1990-2030 the estimated energy usage of renewable sources of the Greek main sectors (industry, 
a

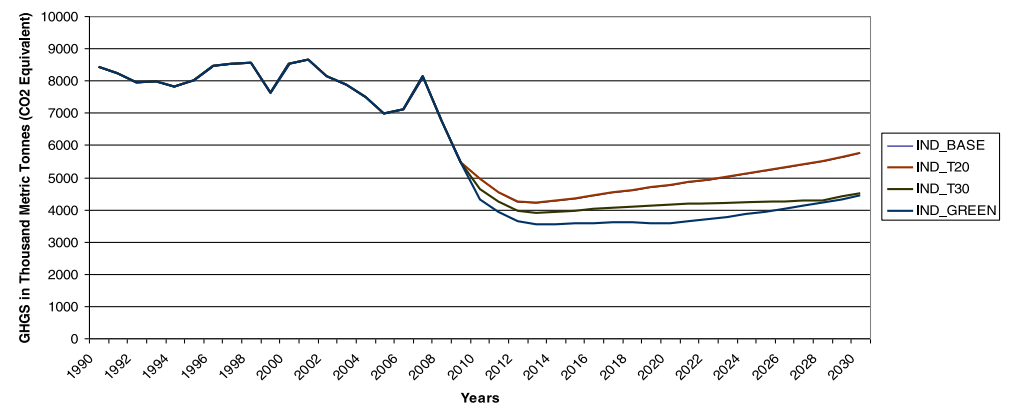

b

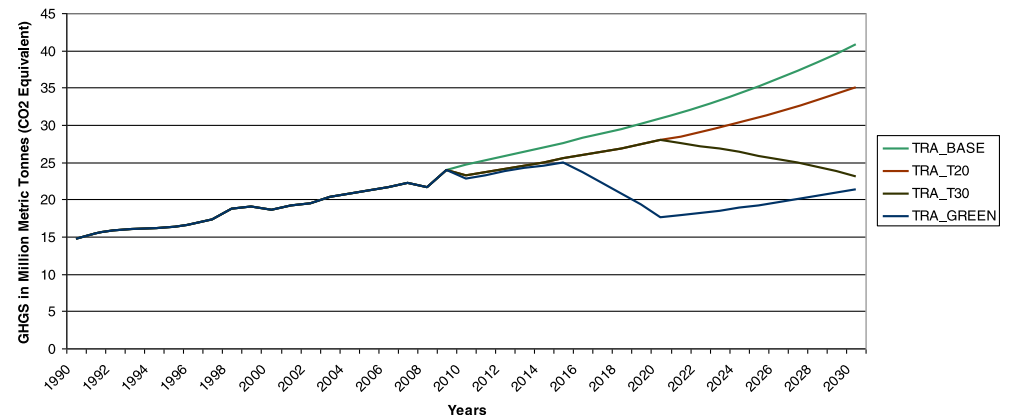

$\mathrm{c}$

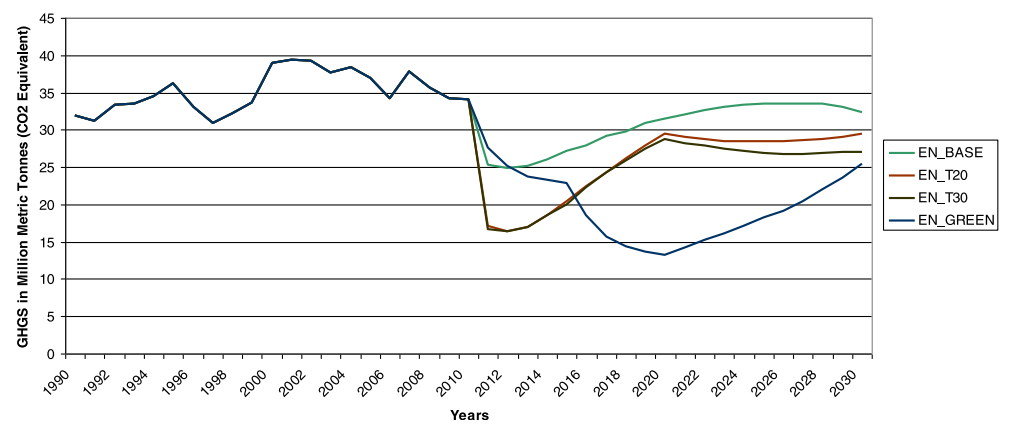

Figure 1 Estimated projections of GHG emissions under the four scenarios (a-industry sector, $\mathbf{b}$-transport sector, c-energy sector).

transport, and energy) alongside with the generated GHG produced. This can be accomplished by creating a composite performance index which can be comparable among the four renewable energy scenarios and among the sectors for the period 1990-2030. As a result, this will enable us to evaluate the efficiency of the renewable energy policy (EREP) based on the future estimates produced using LEAP. ${ }^{5}$

In order to do so, we apply a nonparametric approach known as data envelopment analysis (DEA). DEA is a mathematical programming technique which enables us to evaluate a specific process which is based on the estimation of a benchmark frontier-a relative frontier against which the decision making units (DMUs) are assessed, using specified DMUs' inputs and outputs (Daraio and Simar 2007). Then, the efficiency is calculated as the distance of each DMU from the estimated ("efficient") frontier. In our analysis, we treat as DMU each year which reflects the outcome of the renewable energy policies adopted among the four sectors. 


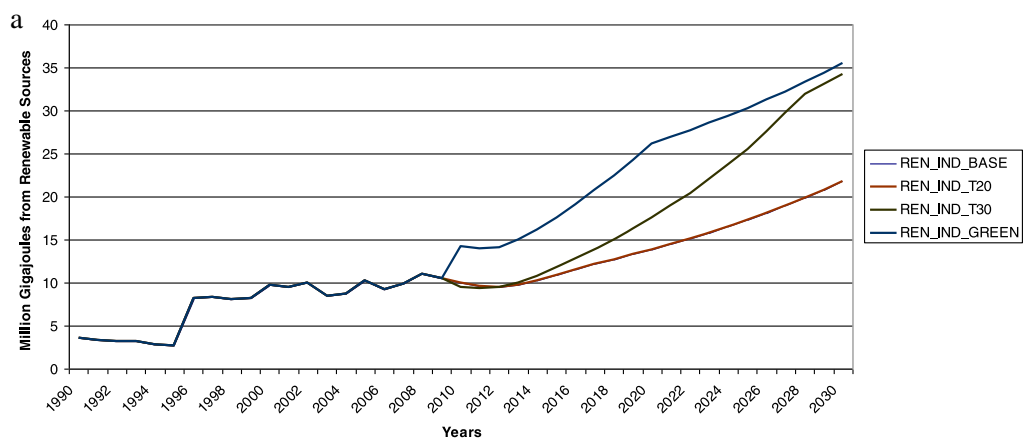

b

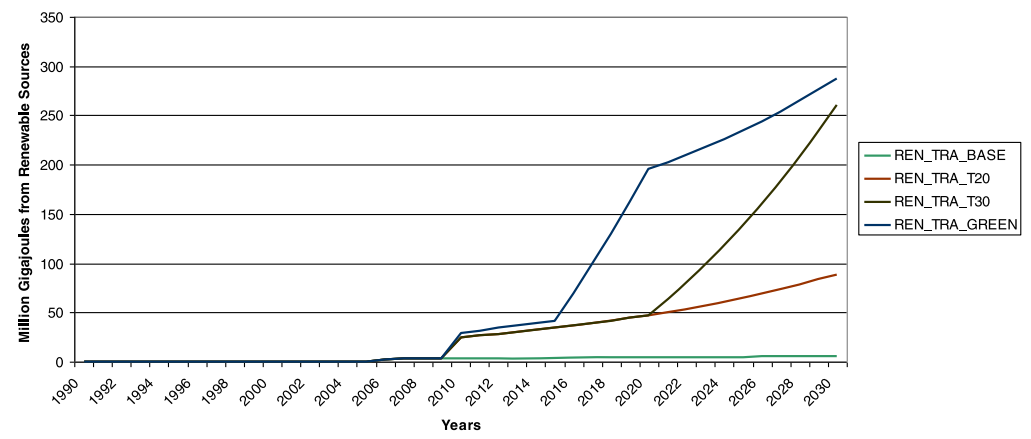

$\mathrm{c}$

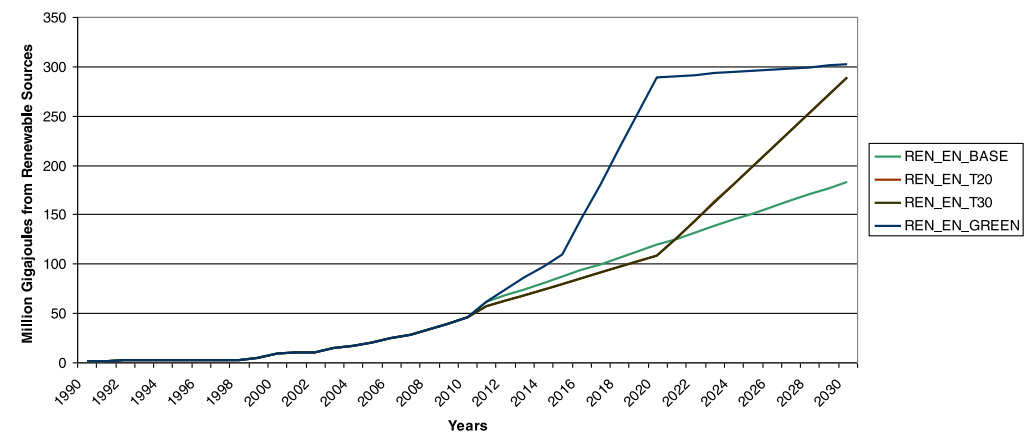

Figure 2 Estimated projections of energy consumption produced by RES under the four scenarios (a-industry sector, $\mathbf{b}$-transport sector, c-energy sector).

Typically, the DEA methodology is applied in a production framework investigating the efficiency of specific inputs to produce specific outputs. However, in our study, we follow a similar approach as the one initiated by Kuosmanen and Kortelainen (2005). They suggest an eco-efficiency indicator which involves the calculation of the ratio of value added (i.e., the good output/GDP) to the environmental damage or pressure index (i.e., the bad output/pollutant), approaching therefore the environmental efficiency from a social point of view rather than from the managerial point of view. Therefore, their proposed index excludes the primary production factors even though they are important cost factors in technical and economic efficiency analysis (Kuosmanen and Kortelainen 2005, p. 64).

In our case, the value added to the Greek economy and society (from the renewable energy policy perceptive) is the energy consumption (measured in million gigajoules) derived from renewable sources. In contrast, the bad output is the greenhouse emissions 
$\left(\mathrm{CO}_{2}, \mathrm{CH}_{4}\right.$, and $\left.\mathrm{N}_{2} \mathrm{O}\right)$ which will be produced in the future (based on the scenarios entered in LEAP) from the sectors of industry, energy, and transport and are the products of bad design, adoption, and implementation of renewable energy policies.

Based on the approach by Koopmans (1951), we can define the efficiency of renewable energy policy in a multiple dimensional Euclidean space. For the purpose of our analysis, let us have Mpollutants (greenhouse emissions- $\mathrm{CO}_{2}, \mathrm{CH}_{4}$, and $\mathrm{N}_{2} \mathrm{O}$ ) measured by the variablesu $=\left(u_{1}, \ldots, u_{m}\right)$ and let $\rho$ denote the energy demand of the three sectors derived only from renewable energy sources (measured in million gigajoules). As a result, we will be able to define the pollution generating technology set as:

$$
T=\left\{\begin{array}{l}
(\rho, \mathbf{u}) \in \mathfrak{R}_{+}^{1+M} \mid \text { the energy consumption derived from renewable sources } \rho \\
\text { can be generated also with damage } \mathbf{u} \text { derived from non-renewable energy sources }
\end{array}\right\}
$$

Expression (1) implies that even though and under the specified energy scenarios, there will be a specific percentage of commitment of energy consumption from renewable sources; however, there will be also pollution generated from energy consumption from nonrenewable sources. Therefore, in our case, the efficiency of the renewable energy policies (EREP) implemented by the Greek government will have the aim to reduce the generated pollution. This efficiency can be represented as:

$$
\operatorname{EREP}_{n}=\frac{\mathrm{P}_{n}}{D\left(\mathbf{U}_{n}\right)}
$$

In ratio (2), $D$ represents the damage function of the Mpollutants in a weighted average indicator represented as:

$$
D(\mathbf{u})=v_{1} u_{1}+v_{2} u_{2}+\ldots+v_{m} u_{m}
$$

Since the problem of a proper weight $(v)$ on the pollutants is crucial, we follow Kuosmanen and Kortelainen (2005) suggesting the benefit-of-the-doubt weighting scheme. This approach applies weights that maximize the relative EREP of the evaluated year and industry in comparison with the maximum attainable EREP. This can be calculated as: ${ }^{6}$

$$
\begin{aligned}
& \max _{v} E R E P_{n}=\frac{\mathrm{P}_{n}}{v_{1} U_{n 1}+v_{2} U_{n 2}+\ldots+v_{M} U_{n M}} \\
& \frac{\mathrm{P}_{1}}{v_{1} U_{11}+v_{2} U_{12}+v_{\mathrm{M}} U_{1 M}} \leq 1 \\
& \frac{\mathrm{P}_{2}}{v_{1} U_{11}+v_{2} U_{22}+v_{\mathrm{M}} U_{2 M}} \leq 1 \\
& \vdots \\
& \frac{\mathrm{P}_{N}}{v_{1} U_{N 1+v_{2}} U_{N 2+v_{M}} U_{N M}} \leq 1 . \\
& v_{1}, v_{2}, \ldots, v_{M} \geq 0
\end{aligned}
$$

Therefore, we use weights $v_{m}(m=1, \ldots, M)$ to maximize the EREP ratio, subject to the condition that the highest attainable efficiency score does not exceed the maximum index value of 1 when the same weights are applied across all other years and industries. As can be observed, the weights are not negative and the efficiency score can take the values between 0 and 1 . As can be realized, the value of 1 indicates an efficient renewable energy policy whereas values below 1 indicate inefficient policies. Furthermore, the 
program in (4) is fractional and is difficult to be solved. However, by following Charnes and Cooper (1962) and Charnes et al. (1978), we can transform the fractional program presented in (4) into a linear program as:

$$
\begin{aligned}
& \min _{v} E R E P_{n}^{-1}=v_{1} \frac{U_{n 1}}{\mathrm{P}_{n}}+v_{2} \frac{U_{n 2}}{\mathrm{P}_{n}}+\ldots+v_{M} \frac{U_{n M}}{\mathrm{P}_{n}} \\
& \text { s.t. } \\
& v_{1} \frac{U_{11}}{\mathrm{P}_{1}}+v_{2} \frac{U_{12}}{\mathrm{P}_{1}}+\ldots+v_{M} \frac{U_{1 M}}{\mathrm{P}_{1}} \geq 1 \\
& v_{1} \frac{U_{21}}{\mathrm{P}_{2}}+v_{2} \frac{U_{22}}{\mathrm{P}_{2}}+\ldots+v_{M} \frac{U_{2 M}}{\mathrm{P}_{2}} \geq 1, \\
& \vdots \\
& v_{1} \frac{U_{N 1}}{\mathrm{P}_{N}}+v_{2} \frac{U_{N 2}}{\mathrm{P}_{N}}+\ldots+v_{M} \frac{U_{N M}}{\mathrm{P}_{N}} \geq 1 \\
& v_{1}, v_{2}, \ldots, v_{M} \geq 0 .
\end{aligned}
$$

For the purpose of our analysis, we apply the distance function approach by Shephard (1970) allowing also for variable returns to scale (VRS) (Banker et al. 1984). Since our analysis is based over a large period of time (1990-2030), it is expected that there will be a lot of variations involved in the demand of energy from renewable sources and variations among the pollutants generated from the consumption of nonrenewable energy sources. According to several authors, the assumption of VRS is more suitable when investigating the impact of changing energy use over time and you expect such variations (Honma and $\mathrm{Hu}$ 2013; Fang et al. 2013).

\section{Results and discussion}

As analyzed previously, we compared for each sector separately the EREP for each year between the four scenarios. More analytically, Figure 3 presents the kernel density plots of the estimated efficiency scores using Gaussian kernels (Silverman 1986).

For the case of the industry sector (Figure 3a), the results reveal that the BAU and TAR20 scenario have identical efficiency distributions. ${ }^{7}$ Furthermore, it appears that there is a bimodal distribution of efficiencies with a first peak around the $45 \%$ level of efficiency and a second peak around the 75\%. The bimodality is also reported for the TAR30 and green scenarios. Again, for both scenarios, there is a first peak at the $45 \%$ level of EREP whereas the second peak for the TAR30 is around the $87 \%$ and that for the green scenario is around 100\%. For the case of transport (Figure 3b), the twin-peak is observed only for the green scenario with one peak around $70 \%$ of efficiency and the second peak around $100 \%$.

Under the BAU scenario, the distribution of the efficiencies of the renewable energy policies over the examined period is platykurtic. This indicates that the efficiency estimates are highly dispersed and their distribution is less clustered around the mean than in a leptokurtic distribution. Similar results can be also viewed for the efficiencies of TAR20 and TAR30. Finally, Figure 3c presents the distribution of efficiency estimates for the Greek energy sector. It appears that under the BAU scenario, the efficiency distribution has three peaks: one around 35\%, a second one around 40\%, and a third one around 55\%. Under the TAR20 and TAR30, the distribution is bimodal with a first peak around $38 \%$ and a second peak of $45 \%$ for TAR20 and 50\% for TAR30. 

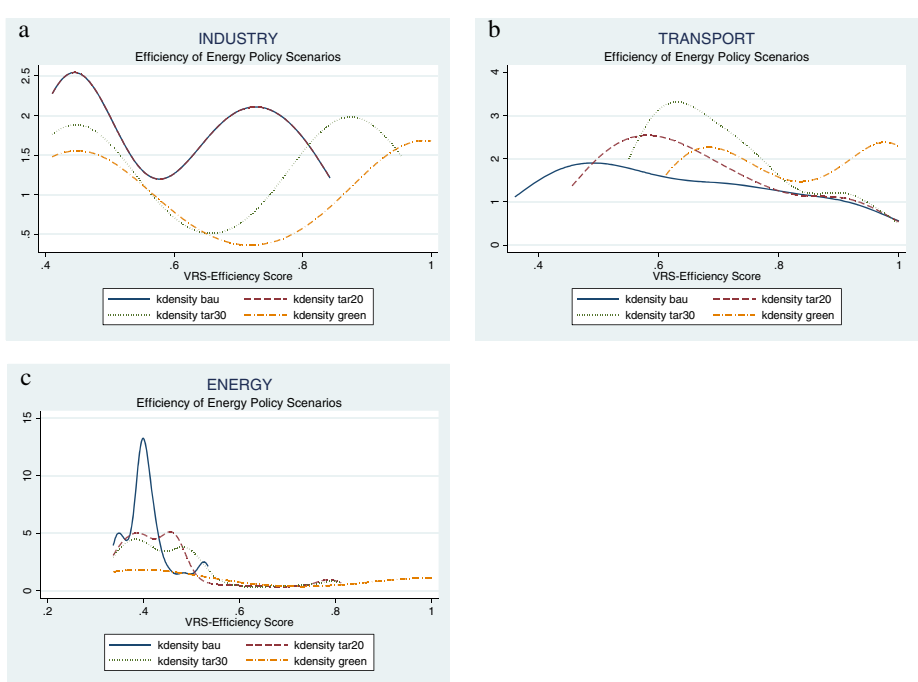

Figure $\mathbf{3}$ Kernel density plots of the estimated efficiency scores (a-industry sector, $\mathbf{b}$-transport sector, c-energy sector).

Similarly, under the green scenario, the distribution of efficiency is platykurtic. Figure 4 presents the efficiency estimates under the four scenarios for the three sectors under examination. When analyzing the industry (Figure 4a), we realize that the efficiency of the renewable energy policies adopted under the BAU and TAR 20 (same line) will decrease over the years. That is, their ability to decrease GHG emissions over the examined period will be weak. As a result, this indicates that the commitments made by the Greek government especially for TAR20 and BAU will be not sufficient to tackle the increased GHG emissions. Under the TAR30, it appears that the EREP will increase after 2024, whereas only under the green scenario, the efficiency of the Greek policy scenarios will be efficient on reducing the projected GHG emissions.

Moreover, Figure 4b represents the efficiency levels for the Greek transport sector. It appears that under the BAU and TAR20, the EREP will decrease over the examined period indicating that under these two scenarios, the Greek government will not succeed on reducing efficiently the GHG emission in the sector of transport. Under the TAR30, the efficiency will increase after 2022 whereas under the green scenario, the efficiency will increase after 2015. Based on those assumptions and for the energy sector, it appears that only under the green scenario the efficiency will increase. Under the BAU scenario, the efficiency will decrease whereas under theTAR20 and TAR30, the efficiencies are in similar efficiency levels.

\section{Conclusions}

The paper analyzes four long-term renewable energy scenarios by using LEAP software for the three Greek sectors. We present the energy consumption estimates from RES and the GHG emissions generated over the period of 1990-2030 for the sectors of industry, transport, and energy. In a second stage analysis, we use DEA methodology in order to evaluate the efficiency of renewable energy commitments on decreasing GHG emissions. The results reveal that the efficiency of renewable energy commitments set by the Greek government under the Law L3851/2010 will not be sufficient to decrease systematically the 


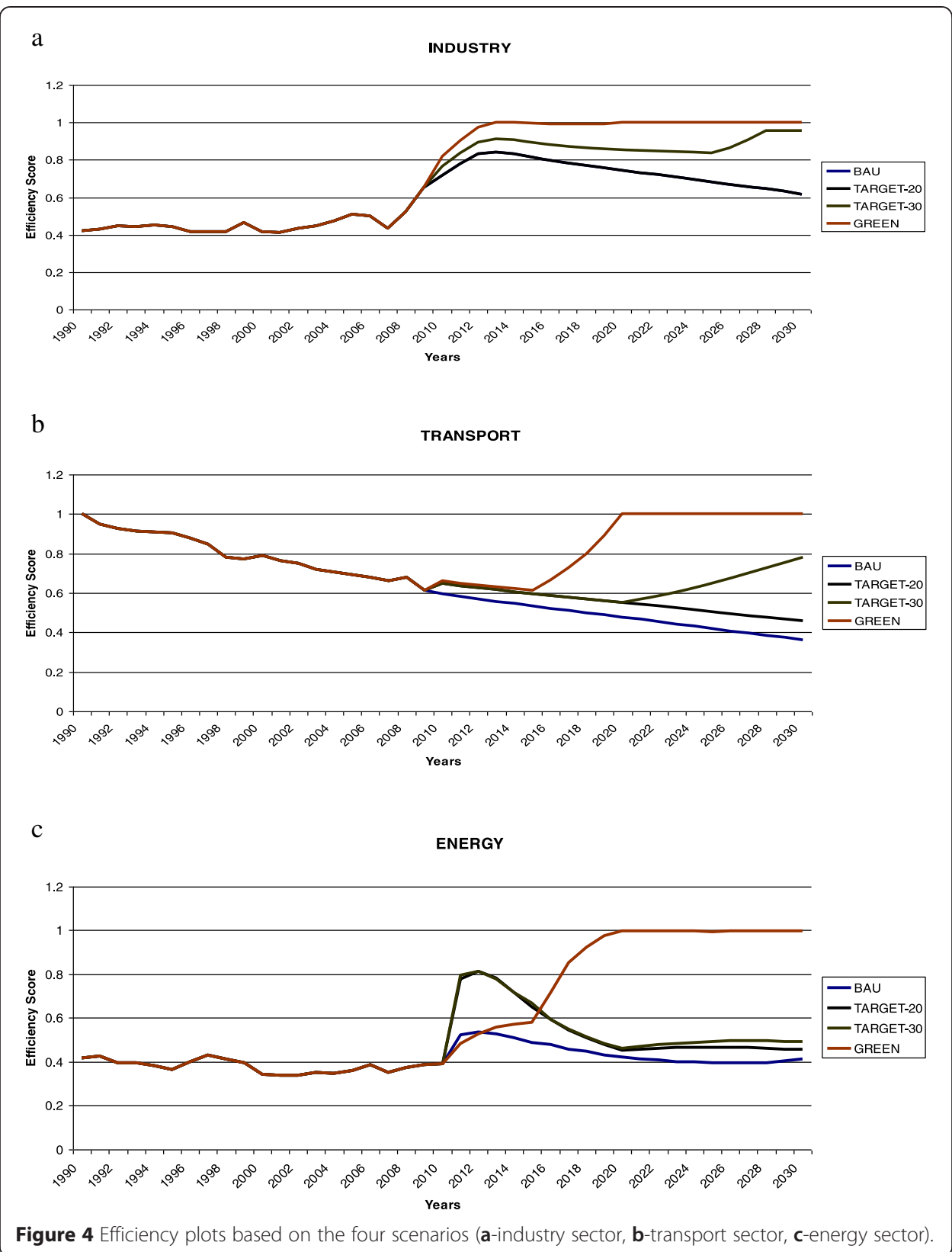

generated GHG emissions over the examined period. In order for the Greek government to have more effective emission-reducing results, it should increase the share of energy consumption produced from renewable resources at least up to $27 \%$ by 2020 . This in turn will decrease significantly more the generated GHG emissions compared to the energy policies which are based on the original commitments set by the Law L3851/2010.

\section{Endnotes}

${ }^{1}$ For some applications of DEA to environmental problems, see Managi and Kaneko (2006), Fukuyama et al. (2011), D' Amato et al. (2012), Nakano and Managi (2012), Halkos and Tzeremes (2013), and Halkos et al. (2015). 
${ }^{2}$ The European targets imply that by 2020, the EU countries' renewable energy penetration in final consumption should be at least by $20 \%$, whereas by 2030 , it should be at least by $27 \%$.

${ }^{3}$ The main scenarios presented in this section have been adopted and are based on the analysis presented by Halkos et al. (2014).

${ }^{4}$ Here, we are interested in the emissions of pollutants. Details on the calculation of control costs of emission reductions may be found in Halkos (1992, 1993, 2010, 2014).

${ }^{5}$ Halkos and Tzeremes (2014a) discuss the effect of electricity consumption from renewable sources on countries' economic growth levels, while Halkos and Tzeremes (2014b) and Halkos (2014) show empirically the effect of countries' compliance with the Kyoto Protocol agreement (KPA) policies.

${ }^{6}$ In our analysis, the letters in uppercase are referring to the observed data, whereas the lowercase letters are referring to theoretical values.

${ }^{7}$ This is due to the fact that the Greek government under the Law L3851/2010 has decided to commit on energy investments from RES only for the sectors of transport, energy, industry, and households. As a result, the BAU energy scenario is identical with the TAR20.

Competing interests

The authors declare that they have no competing interests.

\section{Acknowledgements}

Work in this research has received funding from the "GHGSMETI" program, which takes place within the SYNERGASIA 2011 action and is supported by the European Regional Development fund and Greek National Funds, project number 11SYN-8-118. This paper represents the views of the authors. Finally, we would like to thank the reviewers for the useful and constructive comments made. Any remaining errors are solely the author's responsibility.

Received: 28 January 2015 Accepted: 10 March 2015

Published online: 11 June 2015

\section{References}

Bala BK. Computer modelling of the rural energy system and of CO2 emissions for Bangladesh. Energ. 1997;22:999-1003.

Banker R, Charnes A, Cooper C. Some models for estimating technical and scale inefficiencies in data envelopment analysis. Manage Sci. 1984;30:1078-92.

Bhattacharyya SC, Timilsina GR. A review of energy system models. Int J Energ Sect Manage. 2010;4:494-518.

Cai W, Wang C, Chen J, Wang K, Zhang Y, Lu X. Comparison of CO2 emission scenarios and mitigation opportunities in China's five sectors in 2020. Energ Policy. 2008;36:1181-94.

Carter TR (2007) General guidelines on the use of scenario data for climate impact and adaptation assessment (Task group on data and scenario support for impact and climate assessment-TGICA), Intergovernmental Panel on Climate Change, Version 2, Geneva. Available from: http://www.ipcc-data.org/guidelines/TGICA_guidance_sdciaa_v2_final.pdf.

Charnes A, Cooper W. Programming with linear fractional functionals. Nav Res Log. 1962:9:181-6.

Charnes A, Cooper W, Rhodes E. Measuring the efficiency of decision making units. Eur J Oper Res. 1978;2:429-44.

D' Amato A, Managi S, Mazzanti M. Economics of waste management and disposal: decoupling, policy enforcement and spatial factors. Environ Econ Policy Stud. 2012;14:323-5.

Dagher L, Ruble I. Modeling Lebanon's electricity sector: alternative scenarios and their implications. Energ. 2011;36:4315-26.

Daraio C, Simar L. Advanced robust and nonparametric methods in efficiency analysis. New York: Springer Science; 2007.

Fang CY, Hu JL, Lou TK. Environment-adjusted total-factor energy efficiency of Taiwan's service sectors. Energ Policy. 2013;63:1160-8.

Fukuyama H, Yoshida Y, Managi S. Modal choice between air and rail: a social efficiency benchmarking analysis that considers CO2 emissions. Environ Econ Policy Stud. 2011;13:89-102.

Ghanadan R, Koomey JG. Using energy scenarios to explore alternative energy pathways in California. Energ Policy. 2005;33:1117-42

Giatrakos GP, Tsoutsos TD, Zografakis N. Sustainable power planning for the island of Crete. Energ Policy. 2009;37:1222-38.

Halkos GE. Economic perspectives of the acid rain problem in Europe. University of York, UK: DPhil Thesis; 1992.

Halkos GE. An evaluation of the direct costs of abatement under the main desulphurisation technologies. MPRA Paper 32588. Germany: University Library of Munich; 1993.

Halkos GE. Construction of abatement cost curves: the case of F-gases. MPRA Paper 26532. Germany: University Library of Munich; 2010.

Halkos GE. The Economics of Climate Change Policy: critical review and future policy directions. MPRA Paper 56841 Germany: University Library of Munich; 2014.

Halkos GE, Tzeremes NG. National culture and eco-efficiency: an application of conditional partial nonparametric frontiers. Environ Econ Policy Stud. 2013;15:423-41. 
Halkos GE, Tzeremes NG. The effect of electricity consumption from renewable sources on countries' economic growth levels: evidence from advanced, emerging and developing economies. Renew Sust Energ Rev. 2014a;39:166-73.

Halkos GE, Tzeremes NG. Measuring the effect of Kyoto protocol agreement on countries' environmental efficiency in CO2 emissions: an application of conditional full frontiers. J Prod Anal. 2014b;41:367-82.

Halkos GE, Kevork I, Galani G, Tzeremes P. An analysis of long-term scenarios for the transition to renewable energy in Greece. MPRA Paper 59975. Germany: University Library of Munich; 2014.

Halkos GE, Sundström A, Tzeremes NG (2015) Regional environmental performance and governance quality: a nonparametric analysis. Environ Econ Policy Stud. doi:10.1007/s10018-015-0106-5.

Heaps C (2002) Integrated energy-environment modelling and LEAP, SEl Boston and Tellus Institute, Boston, MA. Available at: http://www.energycommunity.org/default.asp?action=47.

Heaps C (2008) An introduction to LEAP. Available at: http://www.energycommunity.org/documents/LEAPIntro.pdf.

Heaps C, Erickson P, Kartha S, Kemp-Benedict E (2009) Europe's share of the climate challenge. Domestic actions and international obligations to protect the planet. Stockholm Environment Institute. Available at: http://sei-us.org/ Publications_PDF/SEI-EuropeShareOfClimateChallenge-09.pdf.

Honma S, Hu JL. Total-factor energy efficiency for sectors in Japan. Energ Sources Part B. 2013;8:130-6.

Ikonomopoulos A, Tsimoura I, Tsilingiridis G. Projections of green house gas emissions from electricity production in Greece up to 2030. Nicosia, Cyprus: Proceedings of the 4th International Conference on RES and Energy efficiency; 2013.

Islas J, Manzini F, Martinez M. Cost-benefit analysis of energy scenarios for the Mexican power sector. Energ. 2003;28:979-92.

Karapidakis ES, Katsigiannis YA, Georgilakis PS, Thalassinakis E. Generation expansion planning of Crete power system for high penetration of renewable energy sources. Mater Sci Forum. 2011;670:407-14.

Katsigiannis YA, Tsikalakis AG, Karapidakis ES, Katsamaki A. Impact of residential buildings retrofitting actions on the electricity production of Crete Island. Paris, France: Proceedings of the 3rd International Conference on Development, Energy, Environment, Economics; 2012.

Koopmans TC. An analysis of production as an efficient combination of activities. In: Koopmans TC, editor. Activity analysis of production and allocation. Cowles Commission for Research in Economics, Monograph No13. New York: Wiley; 1951.

Kuosmanen T, Kortelainen M. Measuring eco-efficiency of production with data envelopment analysis. J Ind Ecol. 2005;9:59-72.

Law 3851/2010. Accelerating the development of renewable energy sources to address climate change and other provisions relating to the jurisdiction of the Ministry of Environment, Energy and Climate Change. Government Gazette A 85, pp.1753-1780. Available at: http://www.ypeka.gr/LinkClick.aspx?fileticket=qtiW90JJLYs\%3D\&tabid=37 = qtiW90JJLYs\%3D\&tabid $=37$.

Maleviti E, Mulugetta $Y$, Wehrmeyer W. Energy consumption and attitudes for the promotion of sustainability in buildings: the case of hotels. Int J Energ Sect Manage. 2012;6:213-27.

Managi S, Kaneko S. Productivity of market and environmental abatement in China. Environ Econ Policy Stud. 2006; 7:459-70.

Ministry of Environment, Energy and Climate Change (2013). Annual inventory submission of Greece under the convention and the Kyoto Protocol for greenhouse and other gases for the years 1990-2011. Available at: http://www.ypeka.gr/Default.aspx?tabid=285\&language=el-GR.

Moss RH, Edmonds JA, Hibbard KA, Manning MR, Rose SK, van Vuuren DP, et al. The next generation of scenarios for climate change research and assessment. Nature. 2010;463:747-56.

Mulugetta Y, Mantajit N, Jackson T. Power sector scenarios for Thailand: an exploratory analysis 2002-2022. Energ Policy. 2007;35:3256-69.

Nakano M, Managi S. Waste generations and efficiency measures in Japan. Environ Econ Policy Stud. 2012;14:327-39.

Papagiannis G, Dagoumas A, Lettas N, Dokopoulos P. Economic and environmental impacts from the implementation of an intelligent demand side management system at the European level. Energ Policy. 2008;36:163-80.

Park NB, Yun SJ, Jeon EC. An analysis of long-term scenarios for the transition to renewable energy in the Korean electricity sector. Energ Policy. 2013;52:288-96.

Roinioti A, Koroneos C, Wangensteen I. Modeling the Greek energy system: scenarios of clean energy use and their implications. Energ Policy. 2012;50:711-22.

Shephard RW. Theory of cost and production functions. Princeton: Princeton University Press; 1970.

Shin HC, Park JW, Kim HS, Shin ES. Environmental and economic assessment of landfill gas electricity generation in Korea using LEAP model. Energ Policy. 2005;33:1261-70.

Silverman BW. Density estimation for statistics and data analysis. London: Chapman \& Hall/CRC; 1986.

Wiseman J, Biggs C, Rickards L, Taegen E (2011) Scenarios for climate adaptation report. Available at: http://www.vcccar.org.au/sites/default/files/publications/SPCA\%2520REPORT_FINAL_200711_0.pdf

Yophy H, Jeffrey BY, Chieh Yu P. The long-term forecast of Taiwan's energy supply and demand: LEAP model application. Energ Policy. 2011;39:6790-803.

Zhang Q, Weili T, Yumei W, Yingxu C. External costs from electricity generation of China up to 2030 in energy and abatement scenarios. Energ Policy. 2007;35:4295-304. 